\title{
Routing and Wavelength Assignment for Exchanged Hypercubes in Linear Array Optical Networks
}

\author{
Yu-Liang Liu* \\ Department of Computer Science and Information Engineering, Aletheia University, No.32, \\ Zhenli St., Danshui Dist., New Taipei City 25103, Taiwan \\ au4377@au. edu. tw
}

\begin{abstract}
The exchanged hypercube, denoted by EH(s, t), is a new interconnection network obtained by systematically removing links from the hypercube, while preserves many appealing properties. This paper addresses the routing and wavelength assignment for realizing exchanged hypercubes communication patterns on linear array WDM optical networks. By using congestion estimation, we derive a lower bound of the minimum number of required wavelengths, and propose an optimal wavelength assignment algorithm that uses $2^{s+t-1}+$ $\left\lfloor 2^{t} / 3\right\rfloor$ wavelengths.
\end{abstract}

Keywords: WDM optical networks, Routing and wavelength assignment, Exchanged hypercube, Linear array, Congestion.

\section{Introduction}

In a wavelength division multiplexing (WDM for short) optical network, the bandwidth in optical fiber is partitioned into multiple virtual channels, in which different stream of data can be transmitted simultaneously using separate virtual channels. In this context, a virtual channel corresponds to a wavelength. In general, a WDM optical network consists of routing nodes interconnected by point-to-point fiber links. To achieve all-optical communication without optoelectrical conversions at intermediate nodes, end-to-end lightpaths are usually set up between each pair of sourcedestination nodes. A connection or a lightpath in a WDM optical network is an ordered pair of nodes $(S, D)$ corresponding to transmission of a packet from source node $\mathrm{S}$ to destination node $D$.

The primary issue for WDM optical networks is to select a proper path and wavelength satisfying the wavelength-continuity constraint and the distinct wavelength constraint for each connection of a given communication pattern so that the number of used wavelengths is minimized [12-15]. Up to now, there have been some works about routing and wavelength assignments in optical networks [3-5,8,12-14].

The exchanged hypercube is a link-diluted variation of the hypercube nework, proposed by Loh et al [9], with numerous desirable properties, such as lower diameter and better cost effectiveness. Some related works on exchange hypercubes, such as the domination number [6], the connectivity [10], the super connectivity [11], and fault-tolerance measures [7] have been investigated. 
The rest of this paper is organized as follows. In Section 2, we introduce some preliminaries of exchanged hypercubes and the congestion of embedding schemes. In Section 3, a lower bound of the number of required wavelengths for realizing exchanged hypercubes communication patterns on linear arrays is obtained. In Section 4, we propose an embedding scheme and an optimal wavelength assignment algorithm. Finally, we conclude the paper in Section 5.

\section{$2 \quad$ Preliminaries}

In this section, we introduce some preliminaries of exchanged hypercubes and the congestion of embedding schemes.

\subsection{The Exchanged Hypercube}

Let $n$ be a positive integer. The $n$-dimensional hypercube (or $n$-cube for short) $Q_{n}$ is the graph with vertex set $\{0,1\}^{n}$. Two vertices (strings) $u$ and $v$ in $Q_{n}$ are adjacent if and only if they differ in exactly one coordinate. Let $H(u, v)$ denote the Hamming distance between $u$ and $v$, namely the number of coordinates in which $u$ and $v$ are different. Thus two vertices $u$ and $v$ in $Q_{n}$ are adjacent if and only if $H(u, v)=1$.

Let $k>1$ and $u=u_{k-1} \ldots u_{0} \in\{0,1\}^{n}$ be a binary string. We use $u_{j: i}$ to denote the substring $u_{j} u_{j-1} \ldots u_{i}$ of $u$ for $0 \leq i \leq j<k$.

Definition 2.1 ([9]). The vertex set $V$ of exchanged hypercube $\mathrm{EH}(\mathrm{s}, \mathrm{t})$ is the set $\left\{u_{s+t} u_{s+t-1} \ldots u_{0} \mid u_{i} \in\{0,1\}\right.$ for $\left.0 \leq i \leq s+t\right\}$.

Let $u=u_{s+t} \ldots u_{0}$ and $v=v_{s+t} \ldots v_{0}$ be two vertices in $\operatorname{EH}(\mathrm{s}, \mathrm{t})$. There is an edge $(u$,

$v)$ in $\mathrm{EH}(\mathrm{s}, \mathrm{t})$ if and only if $(u, v)$ is in one of the following sets:

$E_{1}=\left\{(u, v) \mid u_{0} \neq v_{0}, u_{i}=v_{i}\right.$ for $\left.0 \leq i \leq s+t\right\}$.

$E_{2}=\left\{(u, v) \mid u_{0}=v_{0}=1, \mathrm{H}(u, v)=1\right.$ with $u_{i} \neq v_{i}$ for some $\left.1 \leq i \leq t\right\}$, and

$E_{3}=\left\{(\mathrm{u}, \mathrm{v}) \mid u_{0}=v_{0}=0, \mathrm{H}(u, \mathrm{v})=1\right.$ with $u_{i} \neq v_{i}$ for some $\left.t+1 \leq i \leq s+t\right\}$.

Let $\mathrm{EH}_{i}(\mathrm{~s}, \mathrm{t})$ be the subgraph of $\mathrm{EH}(\mathrm{s}, \mathrm{t})$ induced by the edges in $E_{i}$ for $\mathrm{i} \in\{1,2,3\}$. Clearly, $\mathrm{EH}(\mathrm{s}, \mathrm{t})$ contains $2^{s+t+1}$ nodes and is a spanning subgraph of hypercube $Q_{s+t+1}$. For $u \in V(\mathrm{EH}(\mathrm{s}, \mathrm{t}))$, if $u_{0}=0$, then the degree of $u$ is $s+1$; otherwise, the degree of $u$ is $t+1$. Fig. 1 depicts $\operatorname{EH}(1,2)$ which is a spanning subgraph of $Q_{4}$. An edge with a label $i$ for $\mathrm{i} \in\{1,2,3\}$ is in edge set $E_{i}$. We can see that each node $u$ in $\operatorname{EH}(1,2)$ with $u_{0}=0$ is of degree 2 and all the other nodes are of degree 3.

\section{Lemma 2.2 ([9]). $\mathrm{EH}(\mathrm{s}, \mathrm{t})$ is isomorphic to $\mathrm{EH}(\mathrm{t}, \mathrm{s})$.}

By Lemma 2.2, hereafter, we may assume without loss of generality that $s \leq t$. 


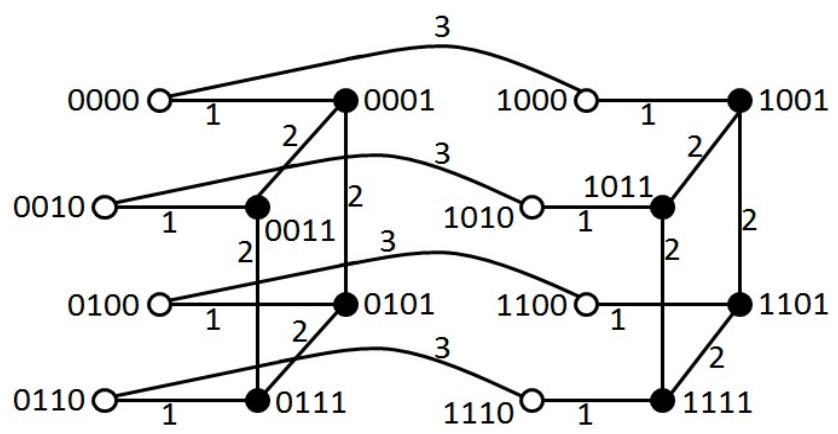

Fig. 1. An exchange hypercube $\mathrm{EH}(1,2)$

Proposition 2.3 ([6]). $\mathrm{EH}_{2}(\mathrm{~s}, \mathrm{t})$ (respectively, $\mathrm{EH}_{3}(\mathrm{~s}, \mathrm{t})$ ) contains $2^{s}$ (respectively, $2^{t}$ ) copies of $Q_{t}$ (respectively, $Q_{s}$ ) in which any two distinct copies of $Q_{t}$ (respectively, $Q_{s}$ ) are disjoint. Moreover, $\mathrm{EH}_{1}(\mathrm{~s}, \mathrm{t})$ forms a perfect matching between nodes in $\mathrm{EH}_{2}(\mathrm{~s}, \mathrm{t})$ and $\mathrm{EH}_{3}(\mathrm{~s}, \mathrm{t})$.

Denote by $Q_{t}^{u_{s+t u+1}}$ for the $Q_{t}$ in $\mathrm{EH}_{2}(\mathrm{~s}, \mathrm{t})$ in which all vertices $u \in Q_{t}$ have the same bits in $u_{s+\mathrm{t}: t+1}$. Similarly, $Q_{s}^{u_{t+1}}$ denotes those $Q_{s}$ in $\mathrm{EH}_{3}(\mathrm{~s}, \mathrm{t})$ for all vertices $u \in Q_{s}$ having the same bits in $u_{t: 1}$. For brevity, $Q_{t}^{u_{s+t i t+1}}$ and $Q_{s}^{u_{t: 1}}$ are also denoted by $Q^{x}{ }_{t}$ and $Q_{s}^{y}$, respectively, where $x$ and $y$ are the decimal values of $u_{s+t: t+1}$ and $\mathrm{u}_{t: 1}$, respectively. Fig. 2(a) and (b) show the two subgraphs $\mathrm{EH}_{2}(1,2)$ and $\mathrm{EH}_{3}(1,2)$, respectively. Note that $\mathrm{EH}_{2}(1,2)$ contains $Q^{0}{ }_{2}$ and $Q^{1}{ }_{2}$ while $\mathrm{EH}_{3}(1,2)$ contains $Q_{1}^{0}, Q^{1}{ }_{1}, Q 2_{1}$, and $Q^{3}$.

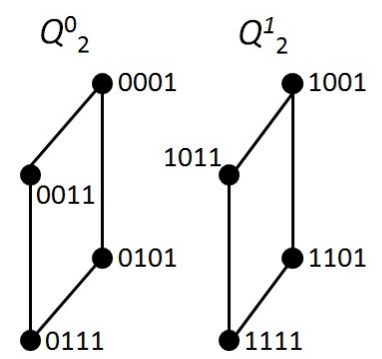

(a) $\mathrm{EH}_{2}(1,2)$

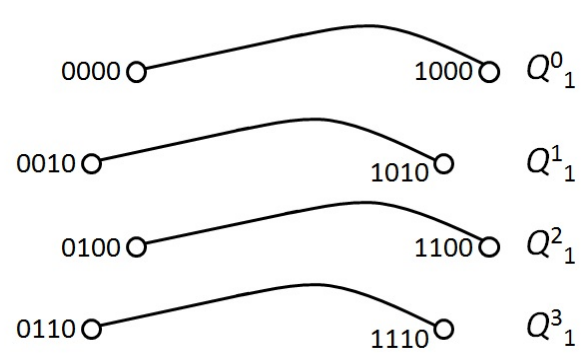

(b) $\mathrm{EH}_{3}(1,2)$

Fig. 2. The two subgraphs of $\mathrm{EH}(1,2)$

\subsection{The Congestion}

Let $G=\left(V_{1}, E_{1}\right)$ be the guest graph and $H=\left(V_{2}, E_{2}\right)$ the host graph, where $\left|V_{1}\right|=\left|V_{2}\right|$. An embedding scheme of $G$ in $H$ is an ordered pair $\Phi=(\Psi, \Omega)$, where $\Psi$ is a bijection from $V_{1}$ to $V_{2}, \Omega$ is a mapping from $E_{1}$ to a set of paths in $H$ such that, for every edge $e=(u, v) \in E_{1}$, there is a path $\Omega(e)$ from $\Psi(u)$ to $\Psi(v)$ in $H$. 
Definition 2.4. The congestion of an edge $e \in E_{2}$ under embedding scheme $\Phi$ of $G$ in $H$, denoted by $c_{e}(G, H, \Phi, e)$, is the number of paths $\Omega\left(e^{\prime}\right)$ for all $e^{\prime} \in E_{1}$ passing through e, namely,

$$
c_{e}(G, H, \Phi, e)=\mid\left\{e^{\prime}: e \in E\left(\Omega\left(e^{\prime}\right)\right), e^{\prime} \in E_{1} \text { and } e \in E_{2}\right\} \mid
$$

The congestion of $G$ in $H$ under $\Phi$, denoted by $c_{p}(G, H, \Phi)$, is defined as:

$$
c_{p}(G, H, \Phi)=\max _{e \in E_{2}} c_{e}(G, H, \Phi, e)
$$

The congestion of $G$ in $H$ under $\Phi$, denoted by $c_{g}(G, H)$, is defined as:

$$
c_{g}(G, H, \Phi)=\min _{\Phi} c_{p}(G, H, \Phi)
$$

Let $\lambda(G, H)$ stand for the number of required wavelengths for realizing communication pattern $G$ on WMN optical network $H$ by embedding scheme $\Phi$. Lemma 2.5 shows that $c_{g}(G, H)$ is a lower bound of $\lambda_{\Phi}(G, H)$.

Lemma $2.5([1,3,12]) \cdot \lambda_{\Phi}(G, H) \geq c_{g}(\mathrm{G}, \mathrm{H})$.

In this paper, we consider that the guest graph is $\mathrm{EH}(\mathrm{s}, \mathrm{t})$ and the host graph is a linear array $L_{n}$, where $n=s+t+1$ and $L_{n}$ is a path of $2^{n}$ nodes. We label the nodes (respectively, the edges) in $L_{n}$ from 1 to $2^{n}$ (respectively, from $e_{1}$ to $e_{2^{n}-1}$ ) in consecutive order. For example, a linear array $L_{3}$ is shown in Fig. 3. Given an embedding scheme, each node $u$ in $V(\mathrm{EH}(\mathrm{s}, \mathrm{t}))$ will be assigned a distinct number in $\left\{1, \ldots, 2^{n}\right\}$. The node assigned number $i$ is then embedded to the node $i$ in $L_{n}$.

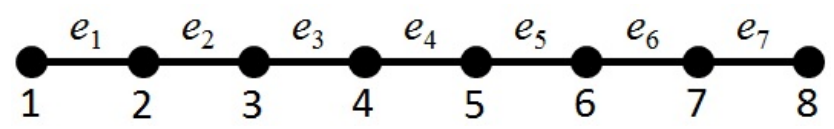

Fig. 3. A linear array $L_{3}$

\section{A Lower Bound of $\lambda_{\Phi}\left(\operatorname{EH}(s, t), L_{n}\right)$}

If $u=u_{s+t} \ldots u_{t+1} u_{t} \ldots u_{1} u_{0}$ is a node in $Q_{t}^{i}\left(0 \leq i \leq 2^{s-1}\right)$ and the decimal value of $u_{t: 1}$ is $j$, then we also use $q^{i, j}$ to denote node $u$. Let $R_{t}^{i}$ stand for the subgraph of EH(s, t) induced by the nodes in $Q_{t}^{i}$ and all nodes in $\mathrm{EH}_{1}$ adjacent to some vertex in $Q_{t}^{i}$.

Lemma 3.1. The congestion of embedding the nodes in $Q_{t}^{i}$ to a linear subarray $L_{t}$ of $L_{n}$ is $2^{t}+\left\lfloor 2^{t} / 3\right\rfloor$.

Property 3.2. If $\Phi$ is an optimal embedding scheme, then $\Phi$ will embed nodes in a subgraph of $\mathrm{EH}(\mathrm{s}, \mathrm{t})$, which is isomorphic to $R_{t}^{i}$, to nodes $1,2, \ldots 2^{t+1}$ of $L_{n}$. 
Lemma 3.3. $\max _{e_{i} ; 1 \leq i \leq 2^{t+1}} \min _{\Phi}\left\{c_{e}\left(E H(s, t), L_{n}, \Phi, e_{i}\right)\right\} \geq 2^{s+t-1}+\left\lfloor 2^{t} / 3\right\rfloor$.

Theorem 3.4. $c_{g}\left(\mathrm{EH}(\mathrm{s}, \mathrm{t}), L_{n}\right) \geq 2^{s+t-1}+\left\lfloor 2^{t} / 3\right\rfloor$.

Lemma 3.5. The number of required wavelengths to realize $\mathrm{EH}(\mathrm{s}, \mathrm{t})$ communication patterns on linear array $L_{n}$ is not less than $2^{s+t-1}+\left\lfloor 2^{t} / 3\right\rfloor$.

Proof. By Lemma 2.5 and Theorem 3.4, the lemma is thus proved.

Q.E.D.

\section{Optimal Wavelength Assignment for Realizing $\mathrm{EH}(\mathrm{s}, \mathrm{t})$ on $L_{n}$}

In this section, we first derive an embedding scheme, and then describe a routing and wavelength assignment algorithm. Let $u=u_{s+t} \ldots u_{t+1} u_{t} \ldots u_{1} u_{0}$ be a node in $V(\mathrm{EH}(\mathrm{s}, \mathrm{t}))$. We partition $V(\mathrm{EH}(\mathrm{s}, \mathrm{t}))$ into eight disjoint subsets as follows:

$$
\begin{aligned}
& S_{1}=\left\{u: u_{t+1}=0 ; u_{1}=0 \text { and } u_{0}=1\right\}, \\
& S_{2}=\left\{u: u_{t+1}=0 ; u_{1}=1 \text { and } u_{0}=1\right\}, \\
& S_{3}=\left\{u: u_{t+1}=1 ; u_{1}=0 \text { and } u_{0}=1\right\}, \\
& S_{4}=\left\{u: u_{t+1}=1 ; u_{1}=1 \text { and } u_{0}=1\right\}, \\
& S_{5}=\left\{u: u_{t+1}=0 ; u_{1}=0 \text { and } u_{0}=0\right\}, \\
& S_{6}=\left\{u: u_{t+1}=1 ; u_{1}=0 \text { and } u_{0}=0\right\}, \\
& S_{7}=\left\{u: u_{t+1}=0 ; u_{1}=1 \text { and } u_{0}=0\right\}, \text { and } \\
& S_{8}=\left\{u: u_{t+1}=1 ; u_{1}=1 \text { and } u_{0}=0\right\} .
\end{aligned}
$$

Clearly, the subgraph induced by $S_{i}(1 \leq i \leq 4)$ comprises $2^{s-1}$ disjoint $(t-1)$-cubes, and the subgraph induced by $S_{i}(5 \leq i \leq 8)$ comprises $2^{t-1}$ disjoint $(s-1)$-cubes. If $s>2$ for the subgraph induced by $S_{m}(1 \leq m \leq 4)$, we denote the $(t-1)$-cube by $Q^{m, i}{ }_{t-1}$ where $i$ $\left(0 \leq i \leq 2^{s-1}-1\right)$ is the decimal value of $u_{s+t: t+2}$, and the node $u$ in $Q^{m, i}{ }_{t-1}$ is represented by $q^{m, i, j}{ }_{t-1}$, where $j\left(0 \leq j \leq 2^{t-1}-1\right)$ is the decimal value of $u_{t: 2}$. Otherwise, if $s=1$, the $(t-1)$-cubeis denoted by $Q^{m, 0}{ }_{t-1}$, and the node $u$ in $Q^{m, 0}{ }_{t-1}$ is denoted by $q^{m, 0, j}{ }_{t-1}$, where $j$ $\left(0 \leq i \leq 2^{t-1}-1\right)$ is the decimal value of $u_{t: 2}$.

Similarly, if $s>2$, for the subgraph induced by $S_{m}(5 \leq m \leq 8)$, we denote the $(s-1)$ cube by $Q^{m, i}{ }_{s-1}$, where $i\left(0 \leq i \leq 2^{t-1}-1\right)$ is the decimal value of $u_{t: 2}$, and the node in $Q^{m, i}{ }_{s-1}$ is represented by $q^{m, i, j}{ }_{s-1}$, where $j\left(0 \leq j \leq 2^{s-1}-1\right)$ is the decimal value of $u_{s+t: t+2}$. Otherwise if $s=1$, the 0 -cube with decimal value $i\left(0 \leq i \leq 2^{t-1}-1\right)$ in substring $u_{t: 2}$ is denoted as $Q^{m, i}$, and the only node in $Q^{m, i}{ }_{0}$ is enoted as $q^{m, i, j}$.

Let $u^{i}=u_{s+t}^{i} \ldots \mathrm{u}_{t+1}^{i} u_{t}^{i} \ldots u_{1}^{i} u_{0}^{i}$ be a node in $S_{i}(1 \leq i \leq 8)$, and let $v=v_{s+t-3} \ldots v_{t+1} v_{t} \ldots$ $v_{1} v_{0}$ be a binary string of length $s+t-2$ with decimal value $x\left(0 \leq x \leq 2^{s+t-2}-1\right)$. If $s>$ 2 , then let $u_{s+t: t+2}^{i}=v_{s+t-3: t-1}$ and $u_{t: 2}^{i}=v_{t-2: 0}$; otherwise, for the case $s=1$, let $u_{t: 2}^{i}=v_{t-2: 0}$. We can find that the nodes $u_{i}(1 \leq i \leq 8)$ form two reversed direction cycles, denoted by $\operatorname{cycle}_{1}(x)$ and $\operatorname{cycle}_{2}(x)$, respectively. Fig. 4 shows the two reversed direction cycles. 


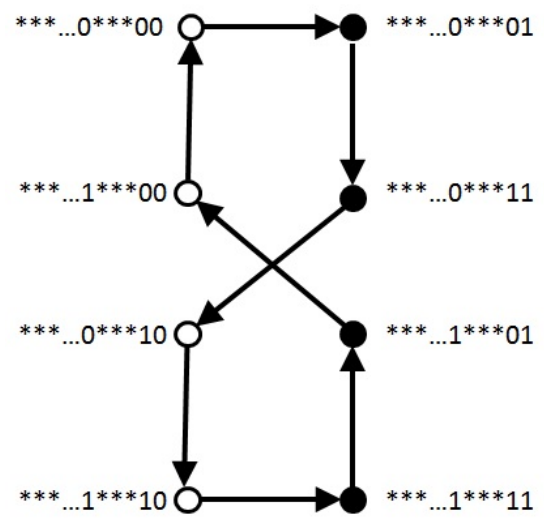

(a) $\operatorname{cycle}_{1}(x)$

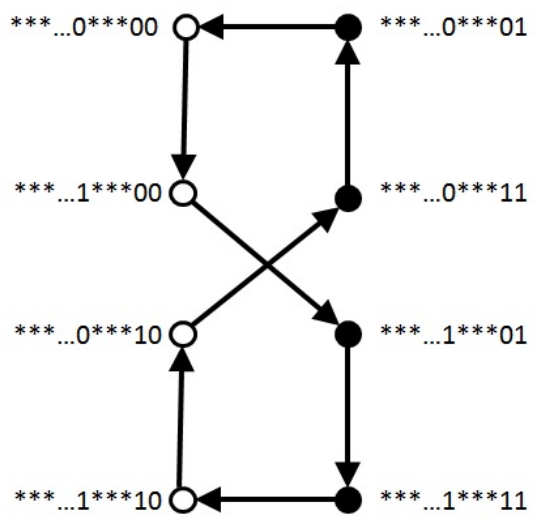

(b) $\operatorname{cycle}_{2}(x)$

Fig. 4. Two reversed direction cycles in $\mathrm{EH}(\mathrm{s}, \mathrm{t})$

An embedding scheme $\alpha$, which assign numbers to the nodes in $\mathrm{EH}(\mathrm{s}, \mathrm{t})$, is shown in Table 1.

Table 1. An embedding scheme $\alpha$

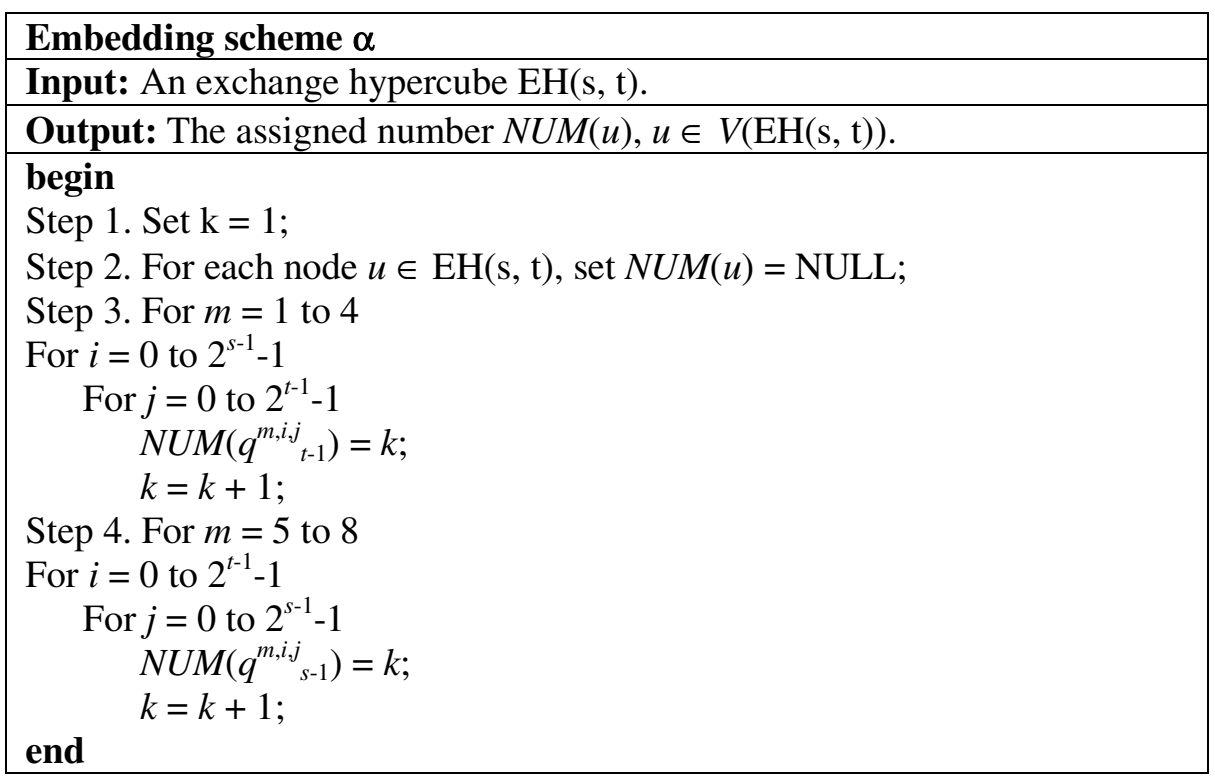

Property 4.1. In the embedding scheme $\alpha$, if $\mathrm{x} \neq \mathrm{y}$ or $m_{1} \neq m_{2}$ or $i_{1} \neq i_{2}$, then the nodes in $\mathrm{Q}^{m 1, i 1}{ }_{x-1}$ and the nodes in $\mathrm{Q}^{m 2, i 2}$ are embedded into two disjoint linear subarrays of $L_{n}$.

Proof. This property is clear from the embedding scheme $\alpha$. 
Fig. 5 shows the numbers assigned to the nodes in $\operatorname{EH}(1,2)$ by the embedding scheme $\alpha$.

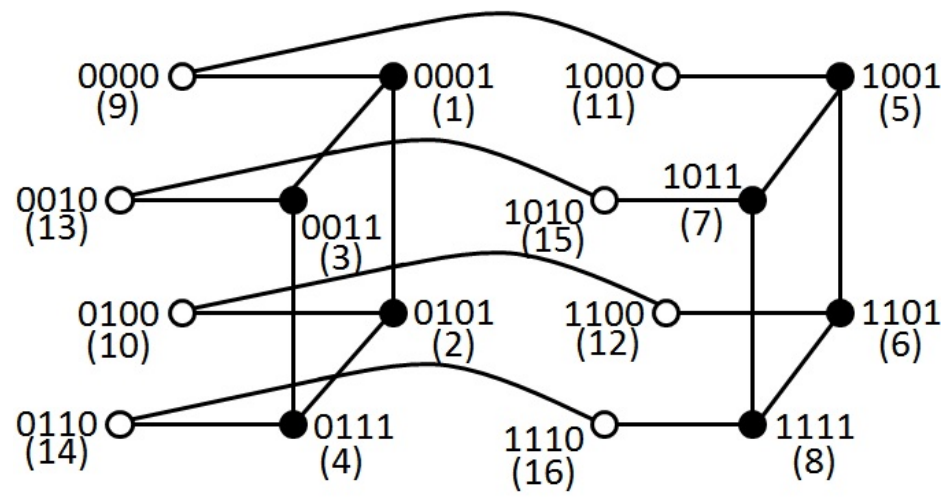

Fig. 5. The numbers assigned to nodes in $\operatorname{EH}(1,2)$

A routing and wavelength assignment algorithm $\beta$ for realizing $\mathrm{EH}(\mathrm{s}, \mathrm{t}) \mathrm{communi}$ cation patterns on $L_{n}$ is shown Table 2 .

Table 2. A routing and wavelength assignment algorithm $\beta$

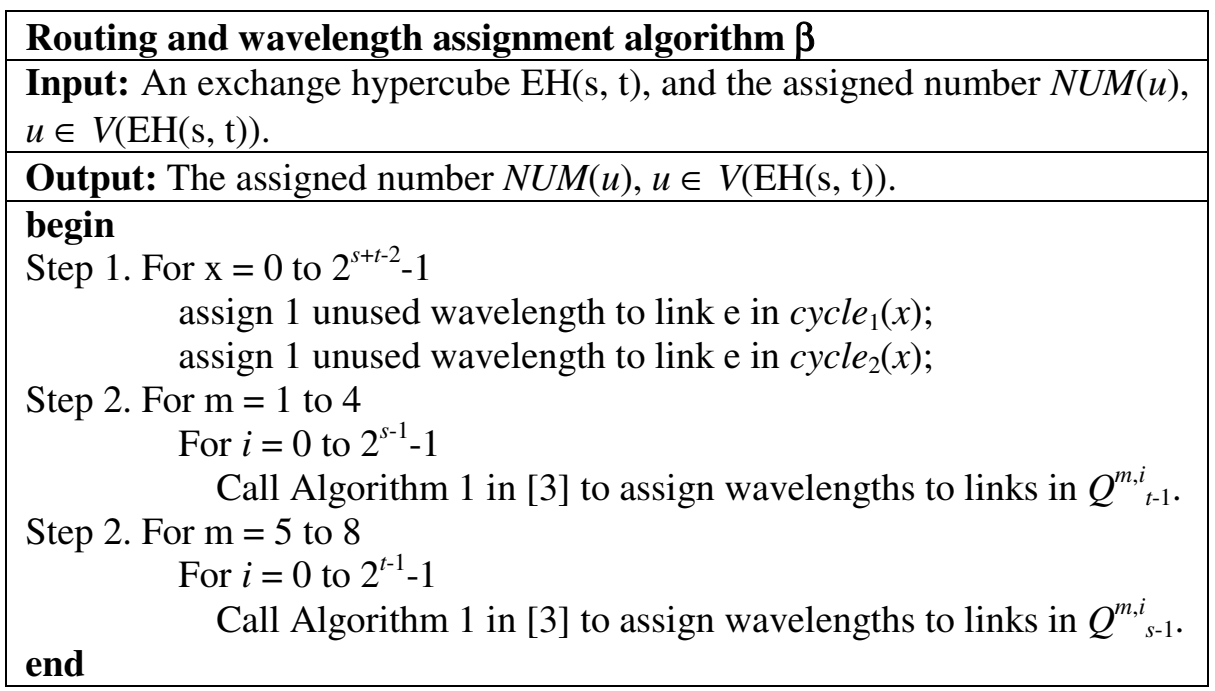

Theorem 4.2. The optimal number of required wavelengths to realize $\mathrm{EH}(\mathrm{s}, \mathrm{t}) \mathrm{com}-$ munication patterns on $L_{n}$ is $2^{s+t-1}+\left\lfloor 2^{t} / 3\right\rfloor$.

Proof. It is clear that Algorithm $\beta$ considers all links in $\mathrm{EH}(\mathrm{s}, \mathrm{t})$. In Step 1, we have that links on $2^{s+t-1}$ directed cycles are assigned wavelengths, and links on each 
directed cycle are assigned 1 unused wavelength. Hence, $2^{s+t-1}$ wavelengths are assigned in this step. In Step 2 (respectively, Step 3), Algorithm 1 is invoked to assign wavelengths to links in $Q^{m, i}{ }_{t-1}$ (respectively, $Q^{m, i}{ }_{s-1}$ ). According to the results in [3], it follows that $\left\lfloor 2^{t} / 3\right\rfloor$ (respectively $\left\lfloor 2^{s} / 3\right\rfloor$ ) wavelengths are required for each $Q^{m, i}{ }_{t-1}$ (respectively, $Q_{s-1}^{m, i}$ ) in Step 2 (respectively, Step 3). By Property 4.1, the wavelengths assigned to links in each $Q^{m, i}{ }_{s-1}$ and $Q^{m, i}{ }_{t-1}$ can be reused, and hence, Steps 2 and 3 require $\left\lfloor 2^{t} / 3\right\rfloor$ wavelengths. It is obvious that Algorithm $\beta$ requires $2^{s+t-1}+$ $\left\lfloor 2^{t} / 3\right\rfloor$ wavelengths. By Lemma 3.5, an optimal wavelength assignment is achieved. This completes the proof.

Fig. 6 shows the wavelengths assigned to the links in $\operatorname{EH}(1,2)$ by the routing and wavelength assignment algorithm $\beta$.

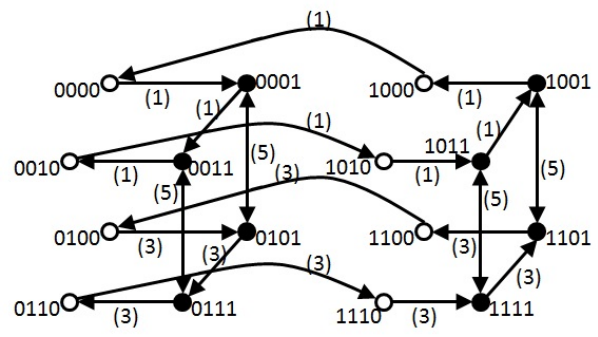

(a)

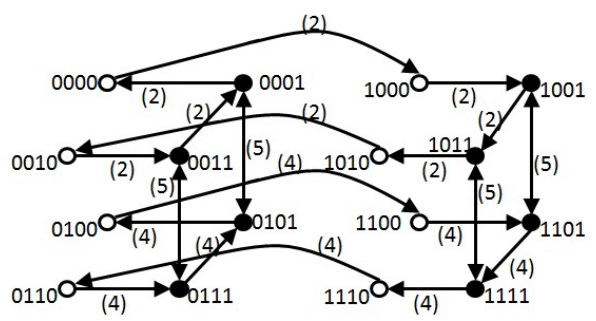

(b)

Fig. 6. The wavelengths assigned to links in $\mathrm{EH}(1,2)$

\section{Concluding Remarks}

In this paper, we study the optimal wavelength assignment for realizing the exchanged hypercube $\mathrm{EH}(\mathrm{s}, \mathrm{t})$ communication patterns on linear array WDM optical network $L_{n}$ by proving that $c_{g}\left(\mathrm{EH}(\mathrm{s}, \mathrm{t}), L_{n}\right) \geq 2^{s+t-1}+\left\lfloor 2^{t} / 3\right\rfloor$. We also design an embedding scheme and a routing and wavelength assignment algorithm which assigns the optimal number of wavelengths.

For the case when $s=t$, the exchanged hypercube is reduce to the dual-cubes [16], and $2^{2 s-1}+\left\lfloor 2^{s} / 3\right\rfloor$ wavelengths are required when the guest network is the dual-cube. For a future research, it is worthwhile to parallelize the wavelength assignment algorithm proposed in this paper, and consider the routing and wavelengths assignment issues for other types of communication patterns, such as, crossed cubes, twisted cubes, recursive circulants, etc.

\section{References}

1. Beauquier, B., Bermond, J.C., Gargano, L., Hell, P., Prennes, S., Vaccaro, U.: Graph problems arising from wavelength routing in all optical networks. In: Proceedings of the Second Workshop in Optics and Computer Science, pp. 76-84 (1997) 
2. Bezrukov, S., Chavez, J., Harper, L., Rottger, M., Schroeder, U.P.: The congestion of ncube layout on a rectangular grid. Discrete Mathematics 213(1-3), 13-19 (2000)

3. Chen, Y., Shen, H.: Routing and wavelength assignment for hypercube in array-based WDM optical networks. Journal of Parallel and Distributed Computing 70, 59-68 (2010)

4. Chen, Y., Shen, H., Liu, F.: Wavelength assignment for realizing parallel FFT on regular optical networks. Journal of Supercomputing 36, 3-16 (2006)

5. Chen, Y., Shen, H., Zhang, H.: Wavelength assignment for directional hypercube communications on a class of WDM optical networks. In: Proceedings of International Conference on Parallel Processing, pp. 288-595 (2007)

6. Klav_zar, S., Ma, M.: The domination number of exchanged hypercubes. Information Processing Letters 114, 159-162 (2014)

7. Li, X.J., Xu, J.M.: Generalized measures of fault tolerance in exchanged hypercubes. Information Processing Letters 113, 533-537 (2013)

8. Libeskind-Hadas, R., Melhem, R.G.: Multicast routing and wavelength assignment in multihop optical networks. IEEE/ACM Transactions on Networking 10(5), 621-629 (2002)

9. Loh, P.K.K., Hsu, W.J., Pan, Y.: The exchanged hypercube. IEEE Transactions on Parallel and Distributed Systems 16(9), 866-874 (2005)

10. Ma, M.: The connectivity of exchanged hypercubes, Discrete Mathematics. Algorithms and Applications 2(2), 51-57 (2010)

11. Ma, M., Zhu, L.: The super connectivity of exchanged hypercubes. Information Processing Letters 111, 360-364 (2011)

12. Yu, C., Yang, X., Yang, L., Zhang, J.: Routing and wavelength assignment for 3-aryncube in array-based optical network. Information Processing Letters 112, 252-256 (2012)

13. Yu, C., Yang, X., Yang, L., Zhang, J.: Routing and wavelength assignment for 3-ary ncube communication patterns in linear array optical networks for $\mathrm{n}$ communication rounds. Information Processing Letters 113, 677-680 (2013)

14. Yuan, X., Melhem, R.: Optimal routing and channel assignments for hypercube communication on optical mesh-like processor arrays. In: Proceedings of The Fifth International Conference on Massively Parallel Processing, pp. 76-84 (1998)

15. Zang, H., Jue, J.P., Bukherjee, B.: A review of routing and wavelength assignment approaches for wavelength-routed optical networks. Optical Network Magazine 1(1), 47-60 (2000)

16. Li, Y., Peng, S., Chu, W.: Efficient collective communications in dual-cube. Journal of Supercomputing 28, 71-90 (2004) 